\title{
Research on intelligent precise pass design algorithm
}

\author{
Zhao Ying \\ School of Economics and Management, Hebei University \\ of Science \&Technology \\ Shijiazhuang,Hebei Province,050018,China \\ Email:zhaoying89321@126.com
}

\section{Gao Yan}

School of Economics and Management, Hebei University of Science \&Technology

Shijiazhuang,Hebei Province,050018,China

Email:gaoyan75@126.com

\author{
Li Guochang \\ School of Economics and Management, Hebei University \\ of Science \&Technology \\ Shijiazhuang,Hebei Province,050018, China \\ Email: lgckdjg@163.com
}

\section{Li Dongmei}

School of Economics and Management, Hebei University of Science \& Technology

Shijiazhuang,Hebei Province,050018,China

Email: 834649353@qq.com

\section{THE ACTUAL ROLLING PROCESS}

formed steel are more and more high in engineering field,the

cross-section geometry shapes become more complex, and the pass design of rolling steel is more difficult. The design method relied on experience and estimation can not meet the requirements of engineering. How to make the system of pass design algorithm stable become the difficulties in the pass design of model steel. Although the researches on cold roll forming theory are more, less theory is used as a guide for pass design. In this paper, a new model of rolling process based on the spline finite strip theory is studied and established, which constructs the Agent structure by using a chain store mode for the shaping of each pass and the deformation of transition zone. The model can realize the stress and strain description of intelligent single-pass roll forming and establish the parameters links between the forming theory and pass design to provide a theoretical basis to deduce accurate pass design.

Keywords : cold-formed steel; pass design; link model; Agent; stress and strain.

\section{INTRODUCTION}

The cold-formed steel have been successfully applied in many fields, which encouraged people to pay more and more attention to the development of new structure. Although now there are more theories of cold roll forming, the real can guide the pass design is very few. The theory that can stably determine the precise pass design algorithm is almost no. The requirements of cold-formed steel are broad and diverse, but the pass design algorithm is difficult to deduct, then they form a tremendous contrast. The bottleneck restricts the manufacture and application of cold-formed steel, at the same time also limits the technology updates and product development in enterprise applying cold-formed steel. How to use the stress-strain theory of the cold forming to solve complex algorithmic problems of pass design is an urgent demand for industrial enterprises.
The rolling process of cold-formed steel is formed by multi-tract pass extrusion, and its stress and strain have a great relationship with strip thickness, elastic modulus, cross-sectional geometry and other factors. Tract

number is determined by cross-section shape, the more complex the cross-section geometry, the more forming tracts. Each tract completes its own forming task, after a series of forming tracts, strip will be rolled into steel. The strip is affected by stress and strain when passing through the forming tract, and strain displacement occurs, after each time of extrusion, the steel morphing into the required section geometry in the interval time is mainly affected by the tensile force, the deformation is small. Therefore, the deformation of the strip is mainly focused on the nodes of the model, the quantitative characterization expression of deformation mainly in the node object. The forming analysis model diagram of strip is shown in figure 1, the deformation expression in forming tracts is shown in formula (1), a dynamic deformation fiction equation in the structure of chain is show in formula (2), (3), transition deformation between tracts and mathematical description is shown in formula (4) .

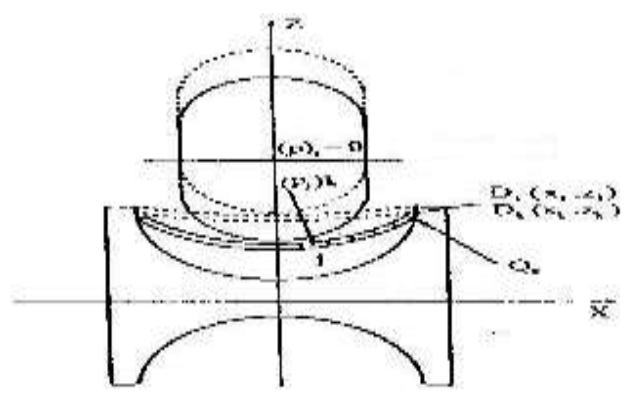

Figure 1. Strip forming analysis model diagram

$\left\{\begin{array}{l}\mathrm{d} \sigma_{y}=\frac{E}{A}\left(-\sigma_{x}^{\prime} \sigma_{y}^{\prime}+2 \gamma B\right) \mathrm{d} \varepsilon x \\ \end{array}\right.$ 


$$
\left.\mathrm{d} \sigma_{\mathrm{x}}=\frac{E}{A}\left(\sigma_{\mathrm{y}}^{\prime 2}+2 B\right)\right) \mathrm{d} \varepsilon x
$$

In above formula,

$$
\begin{aligned}
& \mathrm{A}=C+2\left(1-\mathrm{Y}^{2}\right) B \\
& B=\frac{2 H \sigma^{2}}{9(E-H)}
\end{aligned}
$$

Among these variables, E represents elastic modulus; $\mathrm{H}$ represents material enhancement coefficient; $\gamma$ represents the Poisson's ratio coefficient; $\bar{\sigma}$ represents equivalent stress; $\sigma_{x}^{\prime}$ and $\sigma_{y}^{\prime}$ represent stress deviator.

The analysis of dynamic fiction equation in the rolling process is expanded over time. The initial configuration is changing with the current configuration, the current configuration in $t$ time is the initial configuration in $t+\Delta t$ moment. In this way, each loading step is finished to adjust the material point and stress value,

$$
\begin{aligned}
& { }^{t+\Delta t} x_{i}={ }^{t} x_{i}+\Delta^{t+\Delta t} \delta_{i} \\
& { }^{t+\Delta t} \sigma_{i j}={ }^{t} \sigma_{i j}+\Delta^{t+\Delta t} \sigma_{i j}
\end{aligned}
$$

In this formula, $\Delta^{t+\Delta t} \delta_{i}$ is the incremental displacement calculated loading step from $\mathrm{t}$ to $\mathrm{t}+\Delta \mathrm{t}$ moment.

The corresponding fiction equations is,

$$
\int_{{ }^{t} v}{ }^{t+\Delta t}{ }_{t} S_{i j} \delta_{t}^{t+\Delta t} E_{i j} d v=\delta^{t+\Delta t} W
$$

In this formula, $\delta_{t}^{t+\Delta t}$ represents the virtual displacement from $\mathrm{t}$ to $\mathrm{t}+\Delta \mathrm{t}$ time.

Slow filtration zones between tracts are mainly tensile deformation of the edge of strip, tensile rate of

expression is shown in formula (4)

$$
\varepsilon=\left[\sqrt{\mathrm{L}_{\mathrm{B}}^{2}+2 \mathrm{a}^{2}\left[1-\cos \left(\Delta \theta_{\mathrm{i}}\right)\right]}-\mathrm{L}_{\mathrm{B}}\right] / \mathrm{L}_{\mathrm{B}}
$$

\section{INTELLIGENT PROCESSING FOR DEFORMATION PASSES}

In the expression of describing a single pass, a parameter similar to the elastic modulus, strength coefficient needs to be adjusted, and some expressions in the process of operation need to ignore certain conditions and formula in order to obtain the exact solution,. But because these may cause stress and strain expression can not accurately reflect the actual stress and strain process, which requires supplemented by other methods. The better way is to use the expert experience and knowledge to make decisions, to modify the gap between the theoretical model and the actual model, so that the theoretical model as much as possible to approach the actual model.The specific method is:

1 Set up a corresponding Agent for each deformed finite strip

2 Set the chain node knowledge containers and pointer knowledge container in each Agent;
3 Determine the reasoning rule of knowledge compensation;

4 Construct a reasonable inference mechanism.

The basic framework of Agent is as follows:

Class Agent

Node number Pointer number Record

Node deformation formula

Node knowledge

Pointer---------

Reasoning mechanism

\}

The form of inference rules is as follows:

IF (conditions set) THEN conclusion

For an example, the inference rules of elastic modulus values are as follows:

If $\quad$ Strip thickness $=\mathrm{x} 1 \& \&$ material $=\mathrm{x} 2 \& \&$ forming angle $=\mathrm{x} 3$ then elasticity modulus $=\mathrm{y} 1$

else if Strip thickness $=\mathrm{x} 4 \& \&$ material $=\mathrm{x} 2 \& \&$ forming angle $=\mathrm{x} 3$ then elasticity modulus $=\mathrm{y} 2$

else if Strip thickness $=x 1 \& \&$ material $=x 5 \& \&$ forming angle $=\mathrm{x} 3$ then elasticity modulus $=\mathrm{y} 3$

else if Strip thickness $=x 1 \& \&$ material $=x 2 \& \&$ forming angle $=\mathrm{x} 6$ then elasticity modulus $=\mathrm{y} 4$

The above reasoning rule sets constitute a single Agent knowledge structure, to realize the correction to a single finite deformation theory in a certain time.

\section{PRECISE PASS DESIGN ALGORITHM DERIVATION}

It is a major bottleneck in the field of cold roll forming how to use the roll forming theory to guide the actual pass design. The reason why pass design quality fluctuates largely is that a large gap exists between quantitative algorithm and practical elastic-plastic deformation. Introducing a reasonable theory of cold roll forming to guide pass design is a better method to improve the quality of pass design. Pass design is based on the forming roller diagram of the profiles, and the design of forming roller diagram is derived from the cross section of profiles and analysis of the stress and strain theory in shaping.

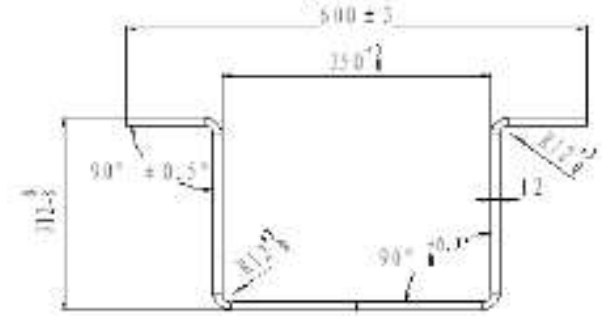

Figure 2. Sectional rawing of cold-formed still 


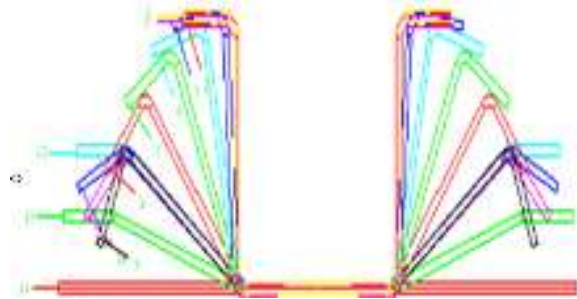

Figure 3. Pass forming roller chart

B1

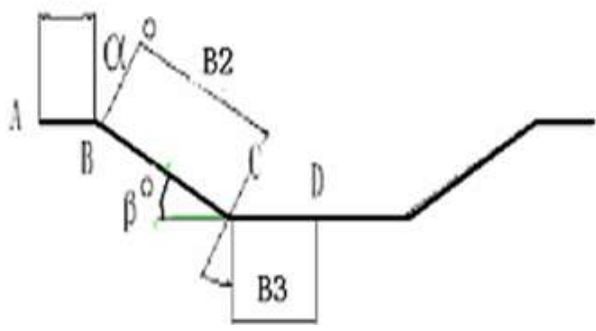

Figure 4. Counting graph of each forming sortie

Excessive deformation may cause tearing strips or rolls easily damaged by excessive force. Too small deformation will increase forming time, reducing molding efficiency.

We use the cold-formed beam as an example to illustrate how to find ideas of precise pass design algorithm. The basic idea of pass design is based on the aimed section geometry, in accordance with the uniform distribution principle of forming force, then design a forming roller diagram gradually modified. According to the diagram, add or remove the curved factor caused by the elastic deformation to calculate pass. Fig.2 shows a cross sectional view of the cold-formed beam, fig. 3 shows the forming roll flower diagram ,fig.4 shows the calculations of each tract. From fig.4, B and C (and its symmetry) point change dramatically, but the rest have no substantially change. The formula of intermediate deformation passes has shown in (5).

$$
\begin{aligned}
& \mathrm{B}_{1}=\mathrm{AB}-\mathrm{R}_{\text {mid }} \times \alpha / 2 \\
& \mathrm{~B}_{2}=\mathrm{BC}-\mathrm{R}_{\text {mid }} \times \alpha / 2-\mathrm{R} \times \beta / 2 \\
& \mathrm{~B}_{3}=\mathrm{CD}-\mathrm{R}_{\text {mid }} \times \beta / 2
\end{aligned}
$$

According to the characteristics of chain structure model, each node corresponds to a pass, and the node of chain model should contain the formula (5). According to the spline finite strip theory, multiple finite strips should be set up in $\mathrm{B}$ and $\mathrm{C}$ points, the relationship between stress and strain has shown in formula (1). d $\sigma \mathrm{x}, \mathrm{d} \sigma \mathrm{y}$, in formula (1) has a close correspondent relationship with $\alpha, \beta$ in formula (5).But this correspondence does not easily said clearly with the expression, adopting artificial intelligence can be the best solutions. Store all in table 3 into the Agent of corresponding nodes in the chain structure, choose $\alpha, \beta$, according to each dox,d $\sigma \mathrm{y}$, then abtain the precise pass algorithm.
TABLE I. BENDING ANGLE Distribution

\section{CONCLUSIONS}

The basis of the pass design is the design of forming roller diagram, the forming roller diagram is based on the profile section geometry and deformation and stress. The deformation and stress depended on the shaping theoretical analysis. According to a reasonable model, use cold roll forming theory to guide pass design to make pass design get rid of the influence of experience, and standardized to a approach contained quantity and expert knowledge, so that the forming roller diagram design and roll surface design can get the stable quality. What said above is a long-awaited thing in engineering field. Although the study of cold roll forming theory and forming theory are more, using the deformation theory to guide the actual pass design is fewer. This paper attempts to integrate theory with practice, using the theory of cold roll forming to quantitatively depict the pass design algorithm, and corrected by artificial intelligence, abtain pass design algorithm with stable quality.

\section{ACKNOWLEDGEMENTS:}

This research is supported by Natural Science Funds of Hebei Province (No. E2011208014); Research and Development Projects of Hebei Province (No. 11457202 d - 8).

\section{REFERENCES}

[1] Zhang Chun-e, Liu Zhen-hua, Zhang Chun-xian,etal. Application research of contact characteristic finite strip in the process of cold roll forming[J].Journal of Hebei University of Science and Technology,2008,29(2):149-151.

[2] Li Guo-chang, Di Shu-gang. Study on the basic framework of intelligent cold roll forming dynamic chain model[J]. Journal of Hebei University of Science and Technology,2012,33(3):218-221.

[3] Zhou Ying.Simulation for Elastic-plastic large deformation of spline finite strip in roll forming process.[D]QIN Huangdao:Mechanical Engineering of Yan Shan University,1996.

[4] Cai Zi-xing, Xu Guang-you.Artificial Intelligence:Principles \& Applications(Fourth Edition)[M].Bei Jing:Tsing Hua University Press,2010.

[5] Yang Xian,Zhang Wen-hua,Li Yao.Computer-aided design of roller pass for cold-formed sill.[J].Shen Yang:Mechanical Design and Manufacturing, 2009 (8) : 122-124.

[6] Zhang Chun-e,Li Guo-chang,Yang Su-fei.Research on the information model and implement strategy of distortion describe of spline fininte strip by using intelligent Agent technology[J] Journal of Hebei University of Science and Technology,2007,28(1):53-56.

[7] Li Guo-chang,Yang Xian.Research on cold roll forming intellectualization methods[J].Han Guan,2013,36(8):25-27.

[8] Li Guo-chang.Research on steel rolling dynamic lincked list model and intelligence Agent deformation analysis[J].Machinery Design \& Manufacture,2013(7):251-252. 
[9] Li Guo-chuang.Research on constructing chain model of cold formed pass deformation analysis and intelligentization[J].Han Guan,2012,35(7):19-21.
[10] Zhang Yu-bin,Li Gu-ochang,Zhao Juan,Sun Wei-ping..Intelligen virtual manufacuring system of cold roll forming roller[J].Hebe Joural of Industrial Science and Technology,2010,27(5):311-313. 